\title{
The role of SRC1 and SRC2 in steroid-induced SDF1 expression in normal and ectopic endometrium
}

\author{
Xiu Shi, Wei Xu, Hui-Hua Dai, Ying Sun and Xiu-Li Wang \\ The Department of Gynecology, The First Affiliated Hospital of Nanjing Medical University (also named Jiangsu \\ Province Hospital), No. 368 North-Jiangdong Road, Gulou, Nanjing, Jiangsu 210036, People's Republic of China \\ Correspondence should be addressed to X-L Wang; Email: xiuli_2266@163.com
}

\begin{abstract}
To compare the expression patterns of steroid receptor coactivators (SRCs) and steroid-induced stromal cell-derived factor 1 (CXCL12 (SDF1)) in normal and ectopic endometrium and to explore the roles of NCOA1 (SRC1) and NCOA2 (SRC2) in the steroid-induced CXCL12 expression in normal and ectopic endometrial stromal cells (ESCs). The NCOA1, NCOA2, NCOA3 (SRC3), and CXCL12 (SDF1) $\alpha$ mRNA levels in normal and ectopic endometrium were analyzed by quantitative real-time PCR. Steroid-induced CXCL12 expression was detected by the ELISA method and the chemotactic activity of conditioned supernatant to monocyte was assessed by the Boyden chamber method before and after the silencing of NCOA1 or NCOA2 with siRNA in normal and ectopic ESCs. The expression of NCOA1 and CXCL12 in ectopic endometrium was significantly greater than that in normal endometrium in the secretory phase. Progesterone $\left(P_{4}\right)$ was able to significantly inhibit estradiol $\left(E_{2}\right)$-stimulated CXCL12 expression in normal and ectopic ESCs. The inhibitory rate of $P_{4}$ in ectopic ESCs at 72 and $96 \mathrm{~h}$ was significantly lower than that in normal ESCs. Silencing of NCOA1 but not NCOA2 significantly reduced the $E_{2}$-induced CXCL12 expression in normal and ectopic ESCs. The ability of $P_{4}$ to inhibit $E_{2}$-induced CXCL12 expression and monocyte chemotaxis in normal and ectopic ESCs was significantly attenuated when NCOA2 was silenced. NCOA1 plays a necessary role in $E_{2}$-induced CXCL12 expression and NCOA2 is required for $P_{4}$ to inhibit the $E_{2}$-induced CXCL12 production in normal and ectopic endometrium.

Reproduction (2014) 147 847-853
\end{abstract}

\section{Introduction}

Endometriosis (EMS) is a complex, estrogen-dependent, and inflammatory disease. Estrogen can be synthesized locally in the endometriotic lesions (Rizner 2009). The immunological factors and angiogenesis play a decisive role in the pathogenesis of EMS (Gazvani \& Templeton 2006). Stromal cell-derived factor 1 (SDF1, also known as CXCL12) is a member of the CXC chemokine family and is a proliferative and chemotactic factor for endothelial cells, lymphocytes, and hematopoietic progenitor cells (Karin 2010, Liu et al. 2011). CXCL12 interacts with CXC chemokine receptor 4 (CXCR4), which is the only physiological receptor for CXCL12. The CXCL12-CXCR4 axis has been shown to have both immune and nonimmune functions, including roles in tissue repair, angiogenesis, invasion, and migration (Cui et al. 2011, Domanska et al. 2013). There is evidence indicating that these mechanisms are also at play in EMS (Ruiz et al. 2010).

CXCL12 has been identified as an estrogen-regulated gene in estrogen receptor (ER)-positive ovarian and breast cancer cells (Hall \& Korach 2003). Estradiol $\left(E_{2}\right)$ enhanced CXCL12 production levels in human endometrial stromal cells (ESCs) in a time- and dosedependent manner (Tsutsumi et al. 2011). Progestins effectively inhibit estrogen-induced CXCL12 expression in human ESCs (Okada et al. 2011). The action of the steroid hormone receptors is modulated in part by members of the p160 family of steroid receptor coactivators (SRCs). The SRC family is composed of three distinct but functionally and structurally related members: SRC1/NCOA1, SRC2/TIF2/GRIP1, and SRC3/ RAC3/ACTR/pCIP/AIB1/TRAM1 (Oñate et al. 1995, $\mathrm{Xu}$ et al. 2009). The SRC family members enhanced the transcriptional activity of a variety of nuclear receptors, including ER and progesterone receptor, and are expressed in a variety of hormone-responsive tissues including the ectopic endometrium (York \& O'Malley 2010, Kumagami et al. 2011). In this study, we observed the expression patterns of SRCs and CXCL12 in normal and ectopic endometrium by quantitative real-time PCR (RT-qPCR) and explored the roles of NCOA1 (SRC1) and NCOA2 (SRC2) in the steroid-induced CXCL12 expression in normal and ectopic ESCs employing siRNA-based gene silencing. 


\section{Materials and methods}

\section{Sources of tissues}

Women with or without EMS, who had not received hormones or gonadotropin-releasing hormone agonist therapy for at least 3 months prior to the study, were recruited after they had provided written informed consent under a study protocol approved by The First Affiliated Hospital of Nanjing Medical University. The ectopic endometrial tissues were obtained from ovarian endometriomata in 16 women with EMS (stage III or IV) who have been identified pathologically and staged according to the revised American Society for Reproductive Medicine (1997) classification. Of them, nine women were in the proliferative phase (days 5-14) and seven in the secretory phase (days 15-28). Normal endometria were acquired from ten healthy women with normal menstrual cycles and no history of EMS and have been identified pathologically. Out of the ten healthy women, five were in the proliferative phase and five in the secretory phase. The ectopic and normal ESCs that were isolated and cultured in vitro successfully were obtained respectively from five ectopic and five normal endometria that were collected in the secretory phase.

\section{Quantitative real-time PCR}

All 16 ectopic and ten normal endometria were analyzed by the RT-qPCR. Primer sequences for NCOA1, NCOA2, NCOA3 (SRC3), and CXCL12 were shown in Table 1. RNA was reverse transcribed with random primers and SuperScript II reverse transcriptase according to Invitrogen's protocol. For analysis, qPCRs were conducted using TaqMan or Power SYBR Green PCR Master Mixes (Applied Biosystems), and samples were amplified with the ABI Prism 7700 sequence detector (Applied Biosystems). The relative mRNA of NCOA1, NCOA2, NCOA3, and $C X C L 12$ was calculated as the ratio of sample gene to the glyceraldehyde 3-phosphate dehydrogenase $(G A P D H)$ gene. All samples were analyzed in triplicate.

\section{Steroid hormone treatment and transient transfection with siRNA}

The ectopic and normal ESCs that were isolated and cultured in vitro successfully were obtained respectively from five ectopic and five normal endometria that were collected in the secretory phase. Human ESCs were purified by the standard enzyme digestion method, as described previously (Ryan et al. 1994, Xiu-li et al. 2009). After passage 0-1, when the ESCs cultured in six-well plates approached $80 \%$ confluence, ESCs were cultured in a medium containing $17 \beta-\mathrm{E}_{2}$ only $\left(10^{-8} \mathrm{~mol} / \mathrm{l} ;\right.$ Sigma $)$ or $17 \beta-\mathrm{E}_{2}\left(10^{-8} \mathrm{~mol} / \mathrm{l}\right)+$ progesterone $\left(\mathrm{P}_{4} ; 10^{-6} \mathrm{~mol} / \mathrm{l} ;\right.$ Sigma). The supernatants were collected 24, 48, 72, and $96 \mathrm{~h}$ after the steroid hormone treatment to quantify CXCL12 (SDF1 $\alpha$ ) expression by the ELISA method. To determine the effect of NCOA1 or NCOA2 silencing on steroid-induced CXCL12 expression, 1 day prior to transfection, $2 \times 10^{5}$ cells were plated into each well of six-well plates and grown overnight in Opti-MEM (Gibco). The next day, cells were transfected with siRNA negative control (Dharmacon Research, Inc., Lafayette, CO, USA), NCOA1 siRNA (Dharmacon Research, Inc.), or NCOA2 siRNA (Dharmacon Research, Inc.) and the final concentration was made up to $100 \mathrm{nM}$ per well using lipofectamine 2000 reagent according to the manufacturer's protocol (Invitrogen).

After $4 \mathrm{~h}$, cells were treated with complete medium. Two days later, media were replaced and $17 \beta-\mathrm{E}_{2}\left(10^{-8} \mathrm{~mol} / \mathrm{l}\right)$ and/or $\mathrm{P}_{4}\left(10^{-6} \mathrm{~mol} / \mathrm{l}\right)$ were added. On day 3 after the steroid hormone treatment, the media were collected to quantify CXCL12 expression. In addition, nuclear proteins were prepared using a kit (Panomics, Redwood, CA, USA) at $48 \mathrm{~h}$ after transfection and assessed by western blotting to verify the reduction in protein expression by siRNA treatments.

\section{CXCL12 ELISA}

CXCL12 in cell culture supernatants was assessed using commercially available sandwich ELISA Kits (Quantikine ELISA Kit, RayBiotech, Inc., Norcross, GA, USA) according to the manufacturer's instructions.

\section{Western blotting analyses}

At $48 \mathrm{~h}$ after transfection with siRNA, nuclear proteins were extracted from ectopic and normal ESCs and were assessed by western blotting to observe the NCOA1 and NCOA2 expression. The protein concentration was determined using a Miro-BCA Protein Assay Kit (Pierce, Rockford, IL, USA). Proteins were separated by SDS-PAGE. Nonspecific sites were saturated by incubating the blots in blocking buffer for $1 \mathrm{~h}$ at room temperature and probed overnight with primary antibodies. After incubation with the appropriate HRPconjugated anti-rabbit or anti-mouse antibody (Amersham Biosciences), detection of specifically bound proteins was carried out by ECL+PLUS according to the manufacturer's protocol (Amersham Pharmacia Biotech) using XL-1 Blue film (Kodak). The density of specific bands was quantified using a densitometer image (Image J, Bethesda, MD, USA). The relative band density was calculated as the ratio of sample to poly(ADP-ribose) polymerase (PARP). The primary antibodies are anti-SRC1 (Santa Cruz Biotechnology), anti-SRC2 (Abcam Ltd., Cambridge, UK), and anti-PARP (Abcam Ltd.).

Table 1 Primer sequences used in real-time PCRs.

\begin{tabular}{lll}
\hline Gene & Forward primer $\left(5^{\prime}-3^{\prime}\right)$ & Reverse primer $\left(5^{\prime}-3^{\prime}\right)$ \\
\hline NCOA1 & TCACCGCAAGAGCTACCAT & CAGCGTCCTCATCTGGTTTC \\
NCOA2 & GCTGGGAGGACCTGGTAAGA & ATTTGACTGAATGCCAATCCT \\
NCOA3 & AGTCTAAATGATGGGCAGTCATG & 193 \\
CXCL12 & TACAGATGCCCATGCCGATT & 124 \\
GAPDH & GGAGTCCACTGGCGTCTTC & TTTTGGCTGTTGTGCTTACTTG \\
\hline
\end{tabular}




\section{Monocyte chemotaxis assay}

The ESCs that were isolated from five ectopic and five normal endometria in the secretory phase and human histiocytic cell line (U937, purchased from the Cell Bank of the Chinese Academy of Science, Shanghai, China) were used for the monocyte chemotaxis assay. U937 cells were treated with $1 \mathrm{mM} 8$-bromo-cAMP for $48 \mathrm{~h}$ to induce a monocyte phenotype and upregulate oligopeptide chemoattractant receptors. For the measurements, 24-well plates assembled with Boyden chambers (0.4-mm pore size polycarbonate membranes) were used. ESCs were placed in the lower chamber and transfected with siRNA. After $4 \mathrm{~h}$, cells were treated with complete medium. Two days later, media was replaced and $17 \beta-\mathrm{E}_{2}$ $\left(10^{-8} \mathrm{~mol} / \mathrm{l}\right)$ and/or $\mathrm{P}_{4}\left(10^{-6} \mathrm{~mol} / \mathrm{l}\right)$ were added. In the upper chamber, $200 \mu$ l suspensions of $U 937$ cells $\left(1 \times 10^{5}\right.$ cells $)$ in PBS were placed. The chambers were then incubated in a humidified $\mathrm{CO}_{2}$ incubator at $37^{\circ} \mathrm{C}$ for $100 \mathrm{~min}$. Nonmigrating cells were removed by washing with PBS and by scraping from the top. The cells migrating across the membrane were fixed, stained, and counted directly. Results are reported as chemotactic index, i.e. cells migrating in response to the test substance divided by cells migrating in siRNA negative control medium.

\section{Statistical analysis}

SPSS 11.0 Software was used in the statistical analysis. All data have been checked by the normality assumption and the homogeneity of variances assumption in one-way ANOVA. Each parameter was presented as mean \pm s.E.M. and compared using one-way ANOVA. The level of significance was set at $P<0.05$.

\section{Results}

\section{RT-PCR analyses of NCOA1, NCOA2, NCOA3, and CXCL12 in normal and ectopic endometrium throughout the menstrual cycle}

As shown in Fig. 1, the NCOA1 level was significantly higher in ectopic endometrium when compared with normal endometrium in the secretory phase. CXCL12 increased statistically in ectopic endometrium when compared with normal endometrium either in the proliferative phase or in the secretory phase. NCOA1, NCOA2, and CXCL12 showed marked cyclic differences in normal endometrium. There was no periodic variation in the expression of NCOA1, NCOA2, NCOA3, and CXCL12 in ectopic endometrium throughout the menstrual cycle. NCOA3 decreased significantly in ectopic endometrium when compared with normal endometrium either in the proliferative phase or in the secretory phase.

\section{Effect of steroid hormones on CXCL12 expression in ectopic ESCS}

Based on the results shown in Fig. 1, we next observed the steroid hormone-induced CXCL12 expression in

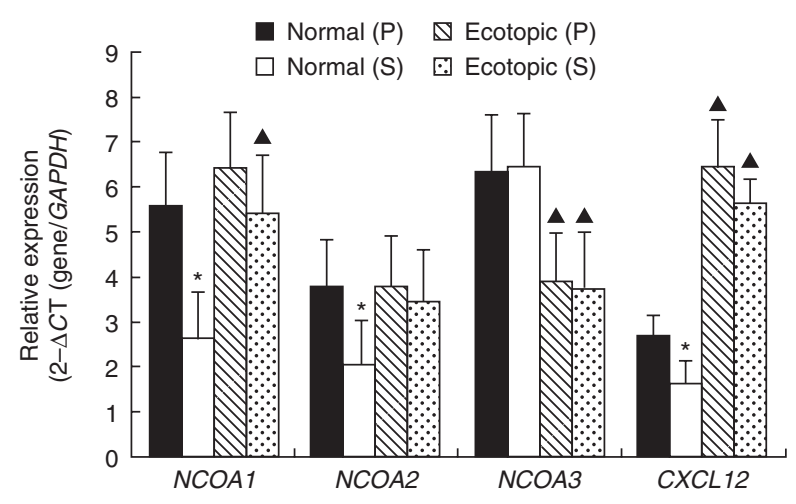

Figure 1 Real-time PCR analyses of NCOA1, NCOA2, NCOA3, and CXCL12 in normal and ectopic endometrium throughout the menstrual cycle. NCOA1, NCOA2, NCOA3, and CXCL12 mRNA of ectopic and normal endometrium obtained from 16 women with EMS and ten normal women without EMS were analyzed by qPCR. Nine women with EMS were in the proliferative $(\mathrm{P})$ phase and seven were in the secretory $(\mathrm{S})$ phase. Five women without EMS were in the $\mathrm{P}$ phase and five were in the $S$ phase. Experiments were performed three times for each sample and each in triplicate. The points represent the mean \pm s.E.M. Relative expression was calculated according to the formula $\left(2-\Delta C \mathrm{~T}\right.$ (gene/GAPDH)). ${ }^{*} P<0.05$ vs $\mathrm{P}$ phase within the same group and $\boldsymbol{\Lambda}, P<0.05$ vs normal endometrium within the same menstrual phase.

ectopic and normal ESCs isolated from five women with EMS and five women without EMS in the secretory phase. CXCL12 in the culture medium was assessed using an ELISA Kit. As shown in Fig. 2, $10^{-8} \mathrm{~mol} / \mathrm{l} \mathrm{E}_{2}$ induced a significant increase in CXCL12 production after $48 \mathrm{~h}$ of culture in normal ESCs (Fig. 2A) and after $24 \mathrm{~h}$ of culture in ectopic ESCs (Fig. 2B) when compared with that in the control, and this effect continued to increase until the end of the study at $96 \mathrm{~h}$. $\mathrm{P}_{4}$ was able to effectively antagonize $\mathrm{E}_{2}$-stimulated $\mathrm{CXCL} 12$ expression in both the groups. At 48, 72, and $96 \mathrm{~h}$, the inhibitory rates were $27.52,33.15$, and $28.31 \%$ respectively in ectopic ESCs and were 31.99, 45.69, and 41.72\% respectively in normal ESCs. The inhibitory rate of $\mathrm{P}_{4}$ in ectopic ESCs was significantly lower than that in normal ESCs at 72 and $96 \mathrm{~h}$.

\section{Effects of SRC silencing on steroid hormone-induced CXCL12 expression in ectopic ESCs}

To investigate the roles of NCOA1 and NCOA2 in the steroid-induced CXCL12 expression, negative control siRNA and siRNA targeting NCOA1 or NCOA2 were transfected into normal and ectopic ESCs respectively, which were subsequently treated with $E_{2}$ only $\left(10^{-8} \mathrm{~mol} / \mathrm{l}\right)$ or $\mathrm{E}_{2}\left(10^{-8} \mathrm{~mol} / \mathrm{l}\right)+\mathrm{P}_{4}\left(10^{-6} \mathrm{~mol} / \mathrm{l}\right)$ for 3 days. As shown in Fig. 3, siRNA effectively reduced NCOA 1 and NCOA2 expression at $48 \mathrm{~h}$ after transfection in ectopic ESCs. Silencing of NCOA1 but not NCOA2 significantly reduced the $\mathrm{E}_{2}$-induced CXCL12 expression in normal (Fig. 4A) and ectopic (Fig. 4B) ESCs. 

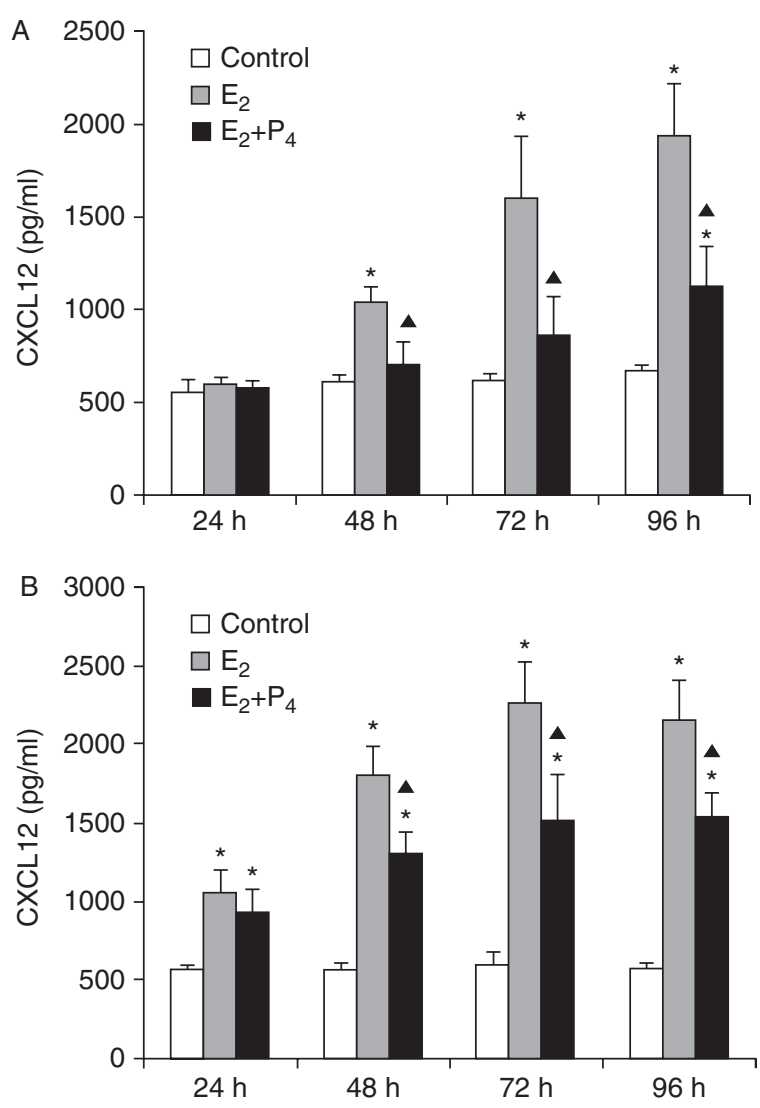

Figure 2 The effects of steroid hormones on CXCL12 expression in normal and ectopic ESCs. Normal (A) and ectopic (B) ESCs were isolated from five women with EMS and five women without EMS in the secretory phase. ESCs were cultured in six-well plates with either control, $E_{2}$ only $\left(10^{-8} \mathrm{~mol} / \mathrm{l}\right)$ or $E_{2}\left(10^{-8} \mathrm{~mol} / \mathrm{l}\right)+\mathrm{P}_{4}\left(10^{-6} \mathrm{~mol} / \mathrm{l}\right)$. ELISA measured CXCL12 concentration in the culture medium at different time points. Experiments were performed three times for each sample and each in duplicate. The points represent the mean \pm S.E.M. $* P<0.05$ vs control group and $\boldsymbol{\Lambda}, P<0.05$ vs $\mathrm{E}_{2}$ group.

There existed a significant difference in CXCL12 expression between NCOA1 silencing and NCOA2 silencing (Fig. 4A and B).

Both in normal (Fig. 5A) and ectopic (Fig. 5B) ESCs treated with $\mathrm{E}_{2}+\mathrm{P}_{4}$, when NCOA1 was silenced, the SDF $1 \alpha$ expression was significantly lower when compared with those treated with $\mathrm{E}_{2}$ and siRNA negative control. When NCOA2 was silenced, the CXCL12 expression in normal (Fig. 5A) and ectopic (Fig. 5B) ESCs treated with $E_{2}+P_{4}$ showed no significant difference when compared with those treated with $E_{2}$ and siRNA negative control. In normal ESCs treated with $\mathrm{E}_{2}+\mathrm{P}_{4}$, the CXCL12 expression after NCOA2 silencing was significantly higher than that after NCOA1 silencing (Fig. 5A). In ectopic ESCs treated with $\mathrm{E}_{2}+\mathrm{P}_{4}$, the CXCL12 expression showed no significant difference between NCOA1 silencing and NCOA2 silencing (Fig. 5B).

\section{Effects of SRC silencing on the chemotactic activity of conditioned supernatant from steroid-treated ectopic ESCs}

Conditioned media from steroid-treated normal and ectopic ESCs that were pre-transfected with siRNA were used for the chemotactic assay in the Boyden chamber. As shown in Fig. 6, whether in normal ESCs (Fig. 6A) or in ectopic ESCs (Fig. 6B) treated with $\mathrm{E}_{2}+\mathrm{P}_{4}$, silencing of NCOA1 has no marked influence on the chemotactic activity of conditioned medium when compared with siRNA negative control. But the chemotactic activity of conditioned medium caused by NCOA2 silencing was significantly higher than that caused by NCOA 1 silencing.

\section{Discussion}

EMS is an estrogen-dependent disease associated with pelvic pain and infertility. The estrogen plays an important role in the planting and growth of ectopic endometrium (Burney \& Giudice 2012). Loss of $P_{4}$ signaling in the endometrium may be a causal factor in the development of EMS, and $\mathrm{P}_{4}$ resistance is commonly observed in women with this disease (Bulun et al. 2010). Tsutsumi et al. (2011) reported that medroxyprogesterone acetate almost completely antagonized the $\mathrm{E}_{2}$-induced CXCL12 production in normal ESCs. In our study, $E_{2}$ stimulated CXCL12 production from normal and ectopic ESCs in a time-dependent manner. Though the $10^{-6} \mathrm{~mol} / / \mathrm{P}_{4}$ cannot completely antagonize the $10^{-8} \mathrm{~mol} / \mathrm{l} \mathrm{E}_{2}$-induced CXCL12 production in this study, the $\mathrm{P}_{4}$ was still able to significantly inhibit $\mathrm{E}_{2}$-stimulated CXCL12 expression in both the normal and ectopic ESCs. Our results showed that the inhibitory rate of $\mathrm{P}_{4}$ in ectopic ESCs was significantly lower than that in normal ESCs at 72 and $96 \mathrm{~h}$ of $\mathrm{E}_{2}$ stimulation. This suggested the existence of $\mathrm{P}_{4}$ resistance in EMS.

One of the mechanisms of $\mathrm{P}_{4}$ resistance in EMS is the perturbed $\mathrm{P}_{4}$ signal transduction. Once bound with
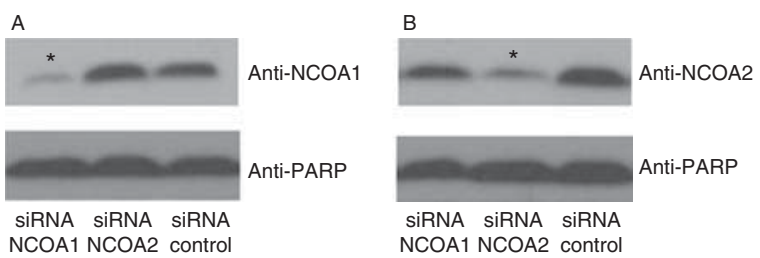

Figure 3 Western blotting image of NCOA1 and NCOA2 expression in ectopic ESCs at $48 \mathrm{~h}$ after transfection with siRNA targeting NCOA1 or NCOA2. At $48 \mathrm{~h}$ after transfection with siRNA, western blotting was used to assess the NCOA1 (A) and NCOA2 (B) protein levels in ectopic ESCs that were isolated from five women with EMS in the secretory phase. The density of specific bands was quantified using a densitometer image. Experiments were performed three times for each sample. The points represent the mean \pm s.E.M. ${ }^{*} P<0.05$ vs siRNA negative control. 

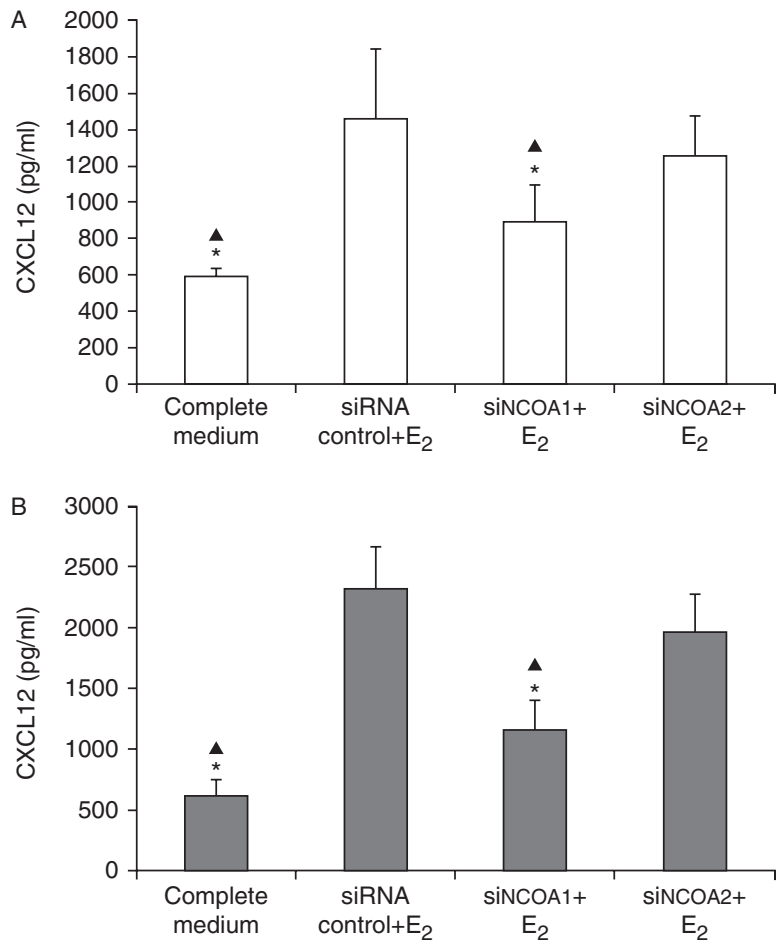

Figure 4 The effect of $N C O A 1$ or $N C O A 2$ silencing on $\mathrm{E}_{2}$-induced CXCL12 expression in normal and ectopic ESCs. siRNA targeting NCOA1 or NCOA2 was transfected into normal (A) and ectopic (B) ESCs isolated from five women with EMS and five women without EMS in the secretory phase respectively, which were subsequently treated with $\mathrm{E}_{2}\left(10^{-8} \mathrm{~mol} / \mathrm{l}\right)$ for 3 days. CXCL12 in the supernatant was detected by the ELISA method. Experiments were performed three times for each sample and each in duplicate. The points represent the mean \pm s.E.M. ${ }^{*} P<0.05$ vs siRNA control $+\mathrm{E}_{2}$ and $\boldsymbol{\Lambda}, P<0.05$ vs $\operatorname{siSRC} 2+\mathrm{E}_{2}$.

ligand-bound nuclear receptors, the SRCs SRC1, SRC2, and SRC3 function to bridge histone acetyl- and methyltransferases to specific enhancer/promoter regions (Johnson \& O'Malley 2012). Several researchers have reported the expression of SRCs in normal endometrium, ectopic endometrium, and ovarian endometriotic tissue detected by immunohistochemistry and semi-quantitative RT-PCR (Suzuki et al. 2010, Kumagami et al. 2011). In this study, the expression patterns and levels of NCOA1, NCOA2, NCOA3, and CXCL12 in normal and ectopic endometrium during the menstrual cycle were characterized using qPCR. The NCOA1 expression in ectopic endometrium was significantly higher than that in normal endometrium in the secretory phase. In nearly all ectopic endometrial glandular and some stromal tissues of ovary, NCOA1 was colocated with ER $\alpha$ (ESR1; Kumagami et al. 2011). Our study adopted the siRNA-based silencing technique to evaluate the roles of NCOA1 and NCOA2 in the steroid hormone-induced expression of CXCL12. The results showed that silencing of NCOA1 but not NCOA2 significantly reduced the $\mathrm{E}_{2}$-induced CXCL12 expression. These suggest that NCOA1 plays a necessary role in $\mathrm{E}_{2}$-induced CXCL12 expression.

We observed that there was no cyclic difference in the expression of the three SRCs in ectopic endometrium throughout the whole menstrual period. Our results are similar to the previous immunohistochemical data (Kumagami et al. 2011). The ability of $\mathrm{P}_{4}$ to inhibit $\mathrm{E}_{2}$ induced CXCL12 expression and monocyte chemotaxis was greatly attenuated compared with $\mathrm{E}_{2}$ stimulation alone when NCOA2 was silenced whether in normal ESCs or in ectopic ESCs, though the CXCL12 expression showed no significant difference between NCOA1 silencing and NCOA2 silencing in ectopic ESCs treated with $E_{2}+P_{4}$. These suggest that NCOA2 is required for $P_{4}$ to inhibit the $\mathrm{E}_{2}$-induced $\mathrm{CXCL} 12$ expression in normal and ectopic endometrium.

Though SRC1 and SRC2 belong to the same family, we found that they play different roles in $\mathrm{E}_{2}$ - and $\mathrm{P}_{4}$-induced CXCL12 expression in normal and ectopic ESCs. NCOA1 is more important for the $\mathrm{E}_{2}$-induced CXCL12 expression and NCOA2 is more important for the activity of $\mathrm{P}_{4}$ to inhibit the $\mathrm{E}_{2}$-induced CXCL12 expression. At the same time, we also observed that expression of NCOA2
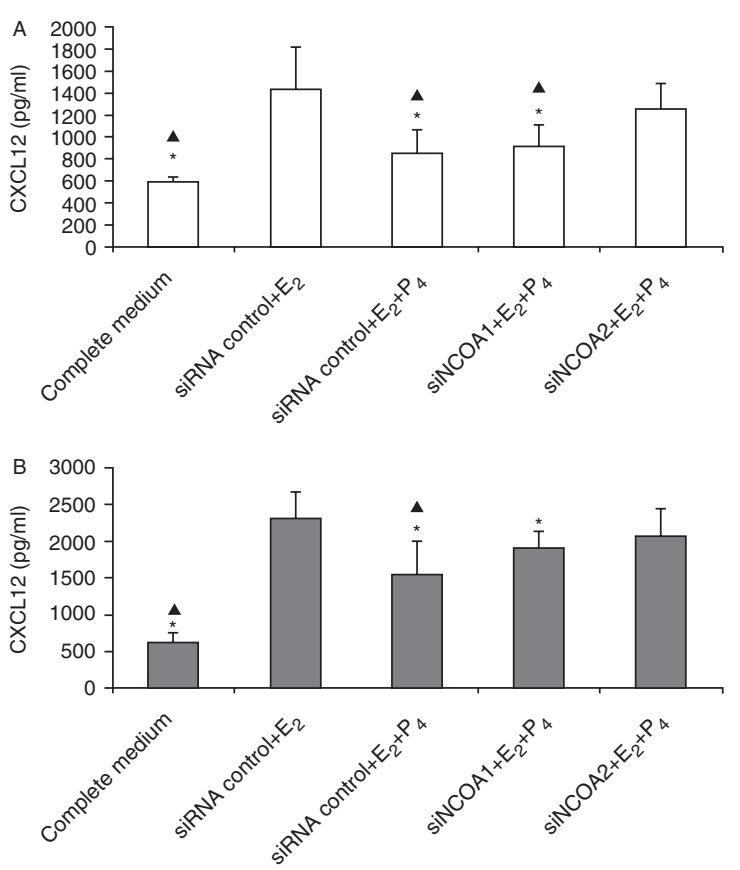

Figure 5 The effect of NCOA 1 or NCOA2 silencing on the inhibitory activity of $\mathrm{P}_{4}$ against $\mathrm{E}_{2}$-induced CXCL12 expression in normal and ectopic ESCs. siRNA targeting NCOA1 or NCOA2 was transfected into normal (A) and ectopic (B) ESCs isolated from five women with EMS and five women without EMS in the secretory phase respectively, which were subsequently treated with $E_{2}$ only $\left(10^{-8} \mathrm{~mol} / \mathrm{l}\right)$ or $E_{2}$ $\left(10^{-8} \mathrm{~mol} / \mathrm{l}\right)+\mathrm{P}_{4}\left(10^{-6} \mathrm{~mol} / \mathrm{l}\right)$ for 3 days. CXCL12 in the supernatant was detected by the ELISA method. Experiments were performed three times for each sample and each in duplicate. The points represent the mean \pm s.E.M. ${ }^{*} P<0.05$ vs siRNA control $+E_{2}$ and $\boldsymbol{\Lambda}, P<0.05$ vs $\operatorname{siSRC} 2+\mathrm{E}_{2}+\mathrm{P}_{4}$. 

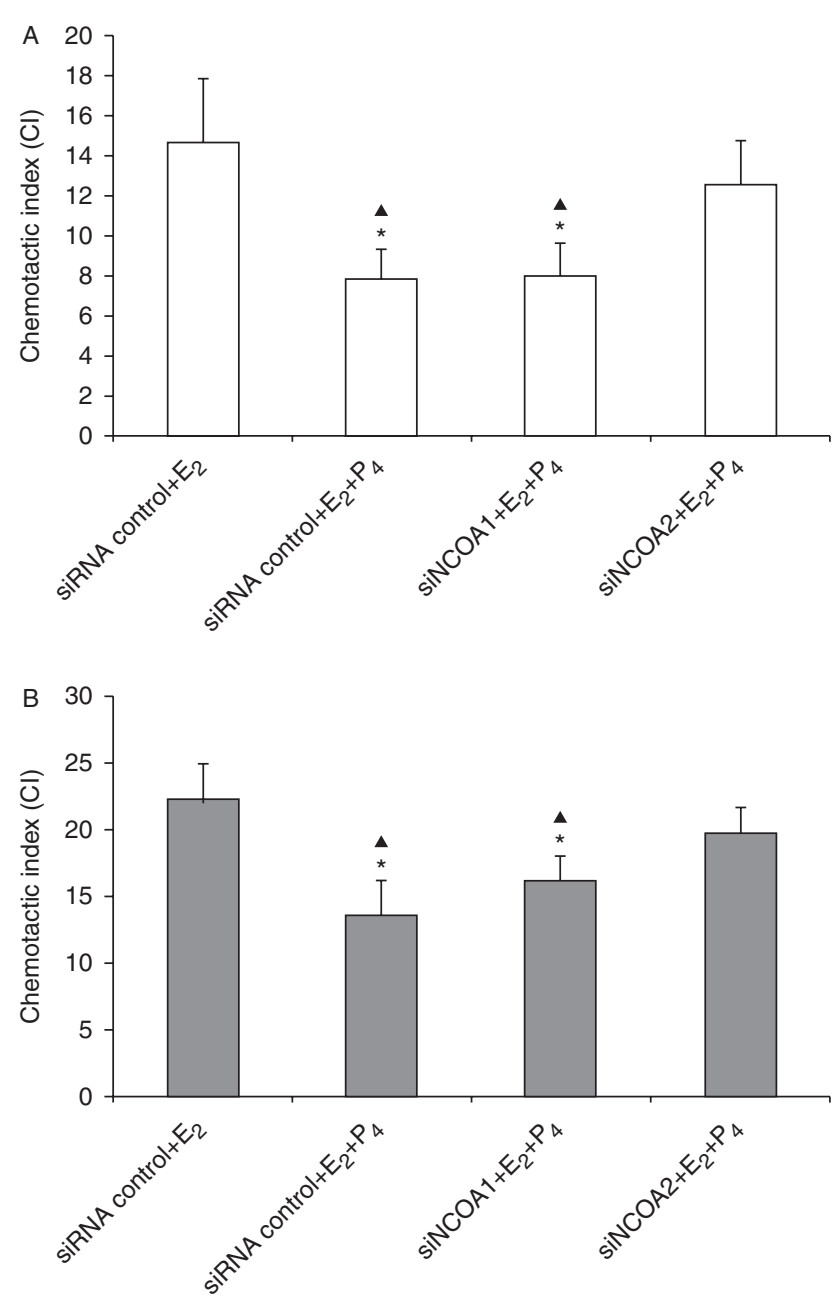

Figure 6 The effect of NCOA1 or NCOA2 silencing on the chemotactic activity of conditioned medium from steroid-treated normal and ectopic ESCs. siRNA targeting NCOA1 or NCOA2 was transfected into normal (A) and ectopic (B) ESCs isolated from five women with EMS and five women without EMS in the secretory phase respectively, which were subsequently treated with $\mathrm{E}_{2}\left(10^{-8} \mathrm{~mol} / \mathrm{l}\right)$ or $\mathrm{E}_{2}+\mathrm{P}_{4}\left(10^{-6} \mathrm{~mol} / \mathrm{l}\right)$ for 3 days. Boyden chambers were used to detect the monocyte chemotaxis of the conditioned medium. Results are reported as chemotactic index $(\mathrm{Cl})$, i.e. cells migrating in response to the test substance divided by cells migrating in siRNA negative control medium. Each point represents the mean \pm s.E.M. $* P<0.05$ vs siRNA control $+\mathrm{E}_{2}$ and $\boldsymbol{\Delta}, P<0.05$ vs siSRC2 $+\mathrm{E}_{2}+\mathrm{P}_{4}$.

mRNA was lower in normal endometrium than in ectopic endometrium in the secretory phase. This is in contrast to the inhibitory activity of $\mathrm{P}_{4}$ in EMS. In fact, there was no significant difference in the NCOA2 mRNA level between the normal and ectopic endometrium in the secretory phase. But the expression of NCOA1 mRNA in ectopic endometrium was significantly higher than that in normal endometrium in the secretory phase. On the other hand, aberrant aromatase activity and defective $E_{2}$ metabolism induced the local high $E_{2}$ environment in EMS (Bulun et al. 2002). These may be the reason for the contradiction between NCOA2 expression and $\mathrm{P}_{4}$ resistance in EMS. Considering the different role of NCOA1 and NCOA2 in steroid-induced CXCL12 expression in ectopic ESCs, we prospect that it may be helpful in improving the $\mathrm{P}_{4}$ resistance in EMS by regulating the NCOA1 and NCOA2 expression.

\section{Declaration of interest}

The authors declare that there is no conflict of interest that could be perceived as prejudicing the impartiality of the research reported.

\section{Funding}

This work was supported by the National Natural Science Foundation of China (no. 81070471 to X-L Wang), the Developing Health Engineering Fund Project of Jiangsu Province of China (no. RC201161 to X-L Wang), and a project funded by the priority Academic Program Development of Jiangsu Higher Education Institutions.

\section{Acknowledgements}

The authors thank the State Key Laboratory of Reproductive Medicine at The First Affiliated Hospital of Nanjing Medical University for excellent technical assistance.

The work was done in the Laboratory of Reproductive Medicine, The First Affiliated Hospital of Nanjing Medical University (also named Jiangsu Province Hospital).

\section{References}

American Society for Reproductive Medicine 1997 Revised American Society for Reproductive Medicine classification of endometriosis: 1996. Fertility and Sterility 67 817-821. (doi:10.1016/S0015-0282(97) 81391-X)

Bulun SE, Gurates B, Fang Z, Tamura M, Sebastian S, Zhou J, Amin S \& Yang S 2002 Mechanisms of excessive estrogen formation in endometriosis. Journal of Reproductive Immunology 55 21-33. (doi:10.1016/ S0165-0378(01)00132-2)

Bulun SE, Cheng YH, Pavone ME, Xue Q, Attar E, Trukhacheva E, Tokunaga H, Utsunomiya H, Yin P, Luo X et al. 2010 Estrogen receptor- $\beta$, estrogen receptor- $\alpha$, and progesterone resistance in endometriosis. Seminars in Reproductive Medicine 28 36-43. (doi:10.1055/s-00291242991)

Burney RO \& Giudice LC 2012 Pathogenesis and pathophysiology of endometriosis. Fertility and Sterility 98 511-519. (doi:10.1016/j.fertnstert.2012.06.029)

Cui K, Zhao W, Wang C, Wang A, Zhang B, Zhou W, Yu J, Sun Z \& Li S 2011 The CXCR4-CXCL12 pathway facilitates the progression of pancreatic cancer via induction of angiogenesis and lymphangiogenesis. Journal of Surgical Research 171 143-150. (doi:10.1016/j.jss.2010.03.001)

Domanska UM, Kruizinga RC, Nagengast WB, Timmer-Bosscha H, Huls G, de Vries EG \& Walenkamp AM 2013 A review on CXCR4/CXCL12 axis in oncology: no place to hide. European Journal of Cancer 49 219-230. (doi:10.1016/j.ejca.2012.05.005)

Gazvani R \& Templeton A 2006 Peritoneal environment, cytokines and angiogenesis in the pathophysiology of endometriosis. Endocrinology 147 1278-1286. (doi:10.1210/en.2005-0790)

Hall JM \& Korach KS 2003 Stromal cell-derived factor 1, a novel target of estrogen receptor action, mediates the mitogenic effects of estradiol in ovarian and breast cancer cells. Molecular Endocrinology 17 792-803. (doi:10.1210/me.2002-0438) 
Johnson A \& O'Malley BW 2012 Steroid receptor coactivators 1, 2, and 3: critical regulators of nuclear receptor activity and steroid receptor modulator (SRM)-based cancer therapy. Molecular and Cellular Endocrinology 348 430-439. (doi:10.1016/j.mce.2011.04.021)

Karin N 2010 The multiple faces of CXCL12 (SDF-1 $\alpha$ ) in the regulation of immunity during health and disease. Journal of Leukocyte Biology $\mathbf{8 8}$ 463-473. (doi:10.1189/jlb.0909602)

Kumagami A, Ito A, Yoshida-Komiya H, Fujimori K \& Sato A 2011 Expression patterns of the steroid receptor coactivator family in human ovarian endometriosis. Journal of Obstetrics and Gynaecology Research 37 1269-1276. (doi:10.1111/j.1447-0756.2010.01509.x)

Liu G, Lu P, Li L, Jin H, He X, Mukaida N \& Zhang X 2011 Critical role of SDF- $1 \alpha$-induced progenitor cell recruitment and macrophage VEGF production in the experimental corneal neovascularization. Molecular Vision 17 2129-2138.

Okada H, Okamoto R, Tsuzuki T, Tsuji S, Yasuda K \& Kanzaki H 2011 Progestins inhibit estradiol-induced vascular endothelial growth factor and stromal cell-derived factor 1 in human endometrial stromal cells. Fertility and Sterility 96 786-791. (doi:10.1016/j.fertnstert.2011.06.048)

Oñate SA, Tsai SY, Tsai MJ \& O'Malley BW 1995 Sequence and characterization of a coactivator for the steroid hormone receptor superfamily. Science 270 1354-1357. (doi:10.1126/science.270.5240.1354)

Rizner TL 2009 Estrogen metabolism and action in endometriosis. Molecular and Cellular Endocrinology 307 8-18. (doi:10.1016/j.mce. 2009.03.022)

Ruiz A, Salvo VA, Ruiz LA, Báez P, García M \& Flores I 2010 Basal and steroid hormone-regulated expression of CXCR4 in human endometrium and endometriosis. Reproductive Sciences 17 894-903. (doi:10.1177/ 1933719110379920)
Ryan IP, Schriock ED \& Taylor RN 1994 Isolation, characterization, and comparison of human endometrial and endometriosis cells in vitro. Journal of Clinical Endocrinology and Metabolism 78 642-649.

Suzuki A, Horiuchi A, Oka K, Miyamoto T, Kashima H \& Shiozawa T 2010 Immunohistochemical detection of steroid receptor cofactors in ovarian endometriosis: involvement of down-regulated SRC-1 expression in the limited growth activity of the endometriotic epithelium. Virchows Archiv 456 433-441. (doi:10.1007/s00428-010-0884-x)

Tsutsumi A, Okada H, Nakamoto T, Okamoto R, Yasuda K \& Kanzaki H 2011 Estrogen induces stromal cell-derived factor 1 (SDF-1/CXCL12) production in human endometrial stromal cells: a possible role of endometrial epithelial cell growth. Fertility and Sterility 95 444-447. (doi:10.1016/j.fertnstert.2010.08.037)

Xiu-li W, Su-ping H, Hui-hua D, Zhi-xue Y, Shi-long F \& Pin-hong L 2009 NF- $\kappa$ B decoy oligonucleotides suppress RANTES expression and monocyte chemotactic activity via NF-kB inactivation in stromal cells of ectopic endometrium. Journal of Clinical Immunology 29 387-395. (doi:10.1007/s10875-009-9274-z)

Xu J, Wu RC \& O'Malley BW 2009 Normal and cancer-related functions of the p160 steroid receptor co-activator (SRC) family. Nature Reviews. Cancer 9 615-630. (doi:10.1038/nrc2695)

York B \& O'Malley BW 2010 Steroid receptor coactivator (SRC) family: masters of systems biology. Journal of Biological Chemistry 285 38743-38750. (doi:10.1074/jbc.R110.193367)

Received 19 January 2014

Accepted 25 February 2014 